\title{
Simultaneous Extrahepatic Portal and Iliac Veins Thrombosis After Abdominal Surgery - A Case Report and Review of Literature
} \author{
Raluca Marginean $5^{*}$, Andreea Varga ${ }^{1}$ \\ 1. Family Medicine Discipline, University of Medicine and Pharmacy Tirgu Mures, Romania \\ 2. Internal Medicine III Discipline, University of Medicine and Pharmacy Tirgu Mures, Romania \\ 3. Victor Babes University of Medicine and Pharmacy Timisoara, Romania \\ 4. Surgery II Discipline, University of Medicine and Pharmacy Tirgu Mures, Romania \\ 5. University of Medicine and Pharmacy Tirgu Mures, Romania
}

Ioan Tilea1, Anca Elena Negovan², Cristina Maria Tatar², Elena Ardeleanu³, Radu Mircea Neagoe4,

Introduction: Extrahepatic portal vein thrombosis (EPVT) is the most frequent cause that leads to portal hypertension in non-cirrhotic patients. This condition is related to systemic and local risk factors (such as inflammatory lesions, injuries to portal venous system by surgery, vascular procedures). Case presentation: A case of extended extrahepatic portal vein thrombosis and simultaneous thrombosis of left common iliac vein and inferior vena cava, appeared after abdominal surgery in a hypertensive, diabetic, 50 y.o. man is presented. An acute episode of abdominal pain was interpreted as an emergency and a surgical (initially laparoscopic and then open) procedure was planned in order to perform an appendectomy. Discharge diagnosis was hemoperitoneum secondary to iatrogenic rupture of sigmoid mesocolon provoked by trocar manipulation. Repeated imaging studies performed later revealed the thrombosis of portal vein with extension into right portal branch associated with superior mesenteric thrombosis and free-floating thrombus into left common iliac vein extended towards inferior vena cava. Surgical manoeuvres are considered as triggers of these thrombotic events. After 4 weeks of parenteral anticoagulation a partial recanalization of thrombi was identified, without bleedings. Conclusions: Acute EPVT needs a carefully management. Case is linked to abdominal surgery and requires prolonged anticoagulation related to simultaneous portal and liac vein thrombosis. Associated conditions (hypertension and diabetes mellitus) must have an appropriate approach. After our knowledge this is the first case published in literature.

Keywords: extrahepatic portal and iliac veins thrombosis, complication of abdominal surgery, anticoagulation, long-term management

Received 04 September 2017 / Accepted 24 November 2017

\section{Introduction}

Extrahepatic portal vein thrombosis (EPVT) - obstruction of the main portal vein with or without extension into the intrahepatic branches - is considered the most common cause which leads to portal hypertension (PHTN) in case of non-cirrhotic patients. Interruption of the portal vein has few clinical consequences, due to two compensatory mechanisms $[1,2]$.

Local and systemic risk factors are involved in the etiology of EPVT; still in 1/3 of patients a combination of thrombotic risk factors are present. Important local factors are cirrhosis, infection, inflammation (diverticulitis, pancreatitis, cholecystitis, inflammatory bowel disease), and malignancy (hepatocellular, gastric, pancreatic cancer). Systemic factors include both genetic deficiencies affecting coagulation and non-genetic conditions (myeloproliferative disorders, polycythemia, pregnancy, use of oral contraceptives). Postoperative portal vein thrombosis (PVT), although rare, can occur after surgical procedures which require the manipulation of major portomesenteric veins (splenectomy) or after laparoscopic procedures (appendec-

* Correspondence to: Raluca Marginean

E-mail: raluca_claudia94@yahoo.com tomy, gallbladder removal, colon or bariatric surgery for morbid obesity) $[1,2,3,4,5,6]$.

Acquired or inherited disorders in the coagulation pathways often provoke PVT. Factor V Leiden deficiency, prothrombin gene G20210A mutations, deficiencies of intrinsic anticoagulation factors are mentioned. Acquired disorders include antithrombin III deficiency, disseminated intravascular coagulation, malnutrition, sepsis, inflammatory bowel disease, liver disease. Rarely, thromboses of the splenic vein has an extension into the portal vein, leading to adjacent inflammatory response (pancreatitis) $[7,8,9]$.

Remarkable, the majority of patients do not present symptoms, while others gradually develop PHTN, varicose veins with a major risk of gastrointestinal massive bleeding.

Spleen enlargement can be observed in rare cases. If EPVT is suspected, non-invasive imaging techniques usually confirm the diagnosis and the presence of solid material into the venous portal lumen $[1,2,8,10,11,12]$.

\section{Case presentation}

The main objective of this paper is to present an extremely rare complication of abdominal surgery and the management of a case of extended PVT in a patient with positive family history of hypercoagulable state or thrombotic 
events (father: thrombophlebitis; brother: procoagulant status); secondary objective is to emphasize the importance of an appropriate medical approach in long-term management of such life-threatening cases in family practice.

A 50-year-old caucasian male, hypertensive, type 2 diabetic insulin-dependent, presented to the emergency medical unit in a secondary-care hospital with an acute episode of abdominal pain related to food abuse. A surgical emergency (acute appendicitis) was considered based on the patient complains, physical examination findings, complete blood count (increased white blood cell count with neutrophilia) and positive CRP; other tests (ALAT, ASAT, serum amylase, urinalysis) were negative. Hyperglycaemia (13.6 $\mathrm{mmol} / \mathrm{l}$ ) was interpreted postprandial. No imaging studies were performed and an emergency appendectomy was considered (initially standard laparoscopic converted to open procedure- pararectus incision). Discharge diagnosis was hemoperitoneum secondary to rupture of sigmoid mesocolon, sustained by a decreased hematocrit level to 35 $\%$. No appendectomy was performed. No anticoagulation regimen was used before and after surgery.

As the epigastric pain did not disappear over the next week after surgery, patient was readmitted and a series of medical tests were performed in order to clarify the etiology of pain. Repeated abdominal and lower-veins ultrasound examinations, thoracic-abdominal CT scans and upper digestive tract endoscopy were performed 12 days after surgery and later on, and the suspicion of EPVT was raised.

Patient was referred to a tertiary care hospital, cardiology unit. Upon admission, patient presents normal physical status, blood pressure, pulse and rest EKG recording within physiological limits and normal bowel transit.

Laboratory data including a basic metabolic panel, complete blood count, presented normal values except INR (1.53), using a standard dose of $2 \mathrm{mg}$ Acenocoumarol daily.

Repeated abdominal ultrasound (Philips HD11XE ultrasound machine) and abdominal CT scan (Siemens Somatom Definition CT scanner) with intravenous contrast media (Iomeron 350/100) revealed the presence of (Fig. 1, Fig. 2, Fig.3):

- Thrombosis into the portal vein with extension into the right portal branch associated with superior mesenteric thrombosis and free-floating thrombus into the left common iliac vein extended towards IVC

- Biliary microlithiasis

- Gallbladder hydrops

- Edematous pancreatitis

As malignancy is one of the leading causes of portal thrombosis, a search for tumoral markers was performed, but the results were negative (alpha fetoprotein $=1.07 \mathrm{UI} /$ $\mathrm{ml}$, CA $19-9<3.0 \mathrm{UI} / \mathrm{ml}$ ).

Patient was tested for inherited disorders in the coagulation pathways, taking into consideration family history and that factor $\mathrm{V}$ Leiden mutation is considered to be the most important thrombophilic predisposing factor for

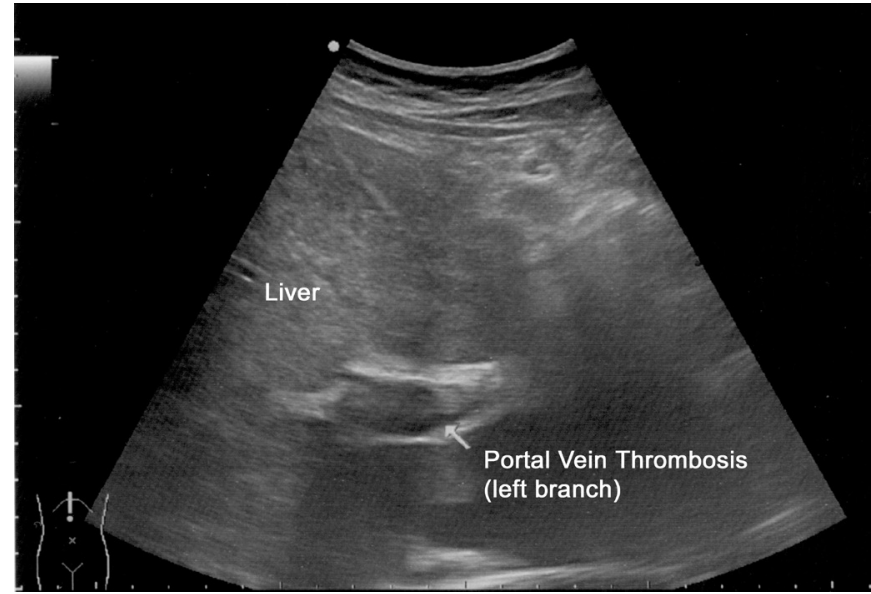

Fig. 1. 2D abdominal ultrasound examination - visualisation of the thrombus in left branch of the portal vein (arrow)

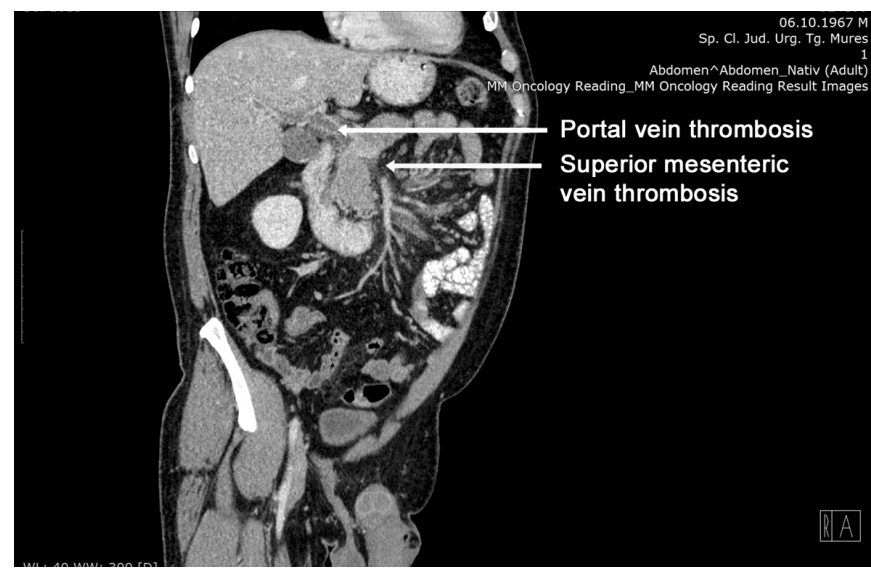

Fig. 2. Contrast-Enhanced abdominal CT examination - visualisation of the thrombus in the portal vein and in the superior mesenteric vein (arrows)

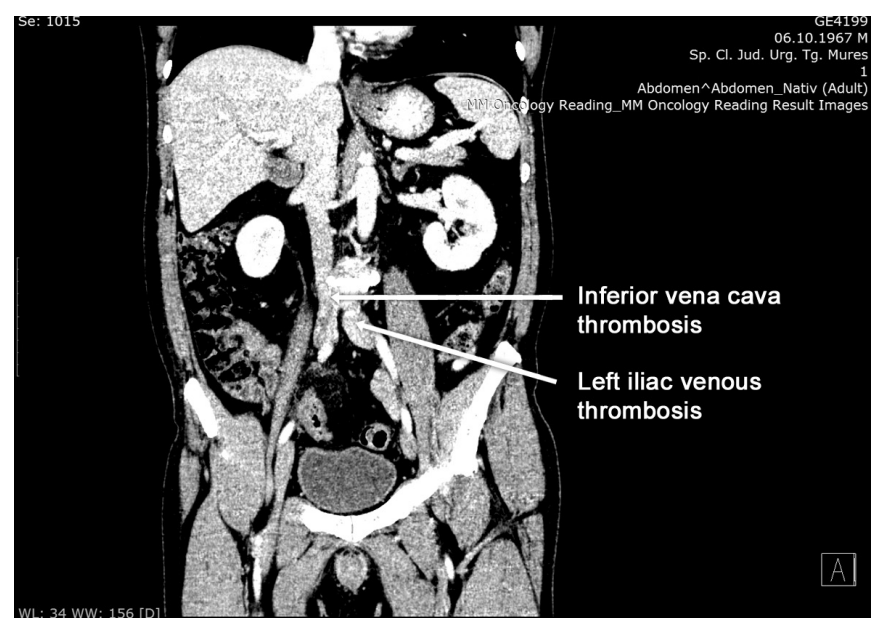

Fig. 3. Contrast-Enhanced abdominal CT examination - visualisation of the thrombus in the inferior vena cava and in the left common iliac vein (arrows)

PVT. Blood samples for a thrombophilic status (protein $\mathrm{C}$ and S deficiency, G20210A mutation in the prothrombin gene, factor $\mathrm{V}$ Leiden mutation, enhanced factor VII activity, JAK2 V617F mutation, VCORK1 -1639G>A gene single nucleotide polymorphism, PAI-1 4G/5G) were negative.

No other possible causes were identified and thus an acute episode of pancreatitis and extensive surgical ma- 
noeuvres were considered as causes of these thrombotic events.

Prolonged parenteral anticoagulation regimen (Enoxaparin $1 \mathrm{mg} / \mathrm{kg}$ body weight b.i.d.) was started, associated with PPI (Pantoprazole $40 \mathrm{mg}$ o.d.), non-selective betablockers (Propranolol $10 \mathrm{mg}$ t.i.d.), a combination of enzymes (Kreon 10000), and adjusted doses of Insulin.

After 4 weeks of parenteral anticoagulation a partial recanalization of portal and iliac veins was confirmed by imaging studies. At discharge medical counselling was given with ambulatory parenteral anticoagulation with $1 \mathrm{mg} / \mathrm{kg}$ body weight b.i.d. Enoxaparin. Patient was scheduled for readmission in 6 weeks for reassessment and switching for oral anticoagulation. Abdominal CT scan with contrast media was performed in order to delineate evolution of extended portal vein thrombosis. A total resolution of the thrombosis from the portal vein and inferior cava vein was observed, while into the common iliac vein was noticed a partial resolution of the thrombus. Oral anticoagulation with adjusted doses of Acenocumarol (for INR: 2.5-3) was started in order to prevent any other thrombotic events. Advices for self-monitoring anticoagulation regimen using a point-of-care device (CoaguCheck ${ }^{\circ} \mathrm{XS}$ Plus Pro System), antihypertensive regimen and self-monitoring of blood sugar levels were offered.

\section{Discussions}

PVT usually represents a rare complication of laparoscopic surgery that can be life threatening if it affects the mesenteric venous arches and leads to mesenteric ischemia or infarction $[3,5,13]$.

Etiology of PVT in non-cirrhotic patients is usually divided into local and systemic factors. Genetic coagulopathies that are considered to be associated with PVT such as factor V Leiden, the prothrombin G20210A mutation, protein $\mathrm{C}$ and $\mathrm{S}$ deficiency, antithrombin III deficiency must be also taken into consideration $[1,2,6,11,12,14,15]$.

Laparoscopic appendectomy may have inherent potential complications. Infection at the surgical sites is the most common associated complication. Other complications include small bowel obstruction, haemorrhage (intraabdominal, hematoma of abdominal wall, scrotal), ileus, stump complications, hernia. In the case of patients with a recent episode of EPVT, the aim of the treatment is to restore flow patency and stop extension of the thrombosis, while in chronic EPVT, portal hypertension management is prior $[1,2]$.

Therapeutic anticoagulation is recommended in stable, non-cirrhotic patients with acute PVT. The recommended duration of treatment is from 6 up to 12 months in order to achieve a stable resolution of thrombosis. Treatment should be provided lifelong in patients known with prothrombotic status, with the goal of maintain a proper recanalization of affected veins $[1,5,10,16,17]$.

Variceal bleeding, the most common and dangerous complication of portal hypertension, can be effectively controlled and prevented using endoscopic therapy [16]. If endoscopic therapy fails to proper manage the bleeding, a transjugular intrahepatic portosystemic shunt, along with another alternative of surgical shunt can be considered [14].

\section{Conclusions}

In this particular case, the diagnosis of appendicitis leads to the decision of a laparoscopic appendectomy. Intraabdominal haemorrhage secondary to the rupture of mesocolon during trocar manipulations direct the decision to a classic open surgery. On abdominal exploration, the appendix was not inflamed; no other specific common surgical pathologies (i.e. acute pancreatitis, cholecystitis, tumours, acute mesenteric ischemia, etc.) were identified; surgical haemostasis of mesosigma was performed. Manoeuvres during laparoscopic appendectomy were considered to be the trigger of extended thrombosis.

Computer-tomography examination with contrast is a valuable and early diagnostic tool of portomesenteric and iliac vein and vena cava thrombosis, which offers the possibility to avoid any kind of bowel gangrene or perforation, or pulmonary embolism, which can lead to peritonitis and worsen the patient's state $[1,2,3]$.

Treatment should be patient-centred, taking into consideration the extension of thrombosis in the vascular system and absence/presence of bowel ischemia or infarction [13]. If a thrombophilic status is confirmed, life-long anticoagulation is mandatory and an extensive screening of relatives must be performed. Patients who suffer from noncirrhotic EPVT can experience new thrombotic events associated with decreased survival, especially if they have a prothrombotic disorder $[1,10,18]$.

Oral anticoagulation treatment (VKA) should be provided long-life for prevention of recurrences or development of chronic thromboembolic pulmonary hypertension, associated with antibiotics in case of infection or systemic inflammatory response syndrome. Anticoagulation usually leads to prevention of new thrombotic events, but the risk of gastrointestinal bleeding should not be neglected.

\section{Conflicts of interest}

None declared.

\section{Acknowledgements}

Patient informed consent was prior obtained for publishing.

This paper is supported by the University of Medicine and Pharmacy of Tirgu Mures and Centrul Medical Topmed Tirgu Mures, Internal Research Grant 6850/15.06.2015.

\section{References}

1. Spaander MC, van Buuren HR, Janssen HL. Review article: the management of non-cirrhotic non-malignant portal vein thrombosis and concurrent portal hypertension in adults. Aliment Pharmacol Ther. 2007; Suppl 2:203-9.

2. Khare R, Sikora SS, Srikanth $G$ et al. Extrahepatic portal venous 
obstruction and obstructive jaundice: approach to management. J Gastroenterol Hepatol. 2005;20(1):56-61.

3. Smalberg JH, Arends LR, Valla DC et al. Myeloproliferative neoplasms in Budd-Chiari syndrome and portal vein thrombosis: a meta-analysis. Blood. 2012;120:4921-28.

4. Webb LJ, Sherlock S. The aetiology, presentation and natural history of extra-hepatic portal venous obstruction. Q J Med. 1979;48(192):62739.

5. Wani ZA, Bhat RA, Bhadoria AS, Maiwall R. Extrahepatic Portal Vein Obstruction and Portal Vein Thrombosis in Special Situations: Need for a New Classification. Saudi J Gastroenterol. 2015;21(3):129-38.

6. Rosenberg JM, Tedesco M, Yao DC, Eisenberg D. Portal Vein Thrombosis Following Laparoscopic Sleeve Gastrectomy for Morbid Obesity. JSLS. 2012;16(4):639-43.

7. Primignani M, Martinelli I, Bucciarelli $P$ et al. Risk factors for thrombophilia in extrahepatic portal vein obstruction. Hepatology. 2005;41(3):603-8.

8. Egesel T, Búyúkasik Y, Dúndar SV et al. The role of natural anticoagulant deficiencies and factor $\vee$ Leiden in the development of idiopathic portal vein thrombosis. J Clin Gastroenterol. 2000;30(1):66-71.

9. Colaizzo D, Amitrano L, Tiscia GL et al. The JAK2 V617F mutation frequently occurs in patients with portal and mesenteric venous thrombosis. J Thromb Haemost. 2007;5(1):55-61.

10. Janssen H,Wijnhoud A, Haagsma $E$ et al. Extrahepatic portal vein thrombosis: aetiology and determinants of survival. Gut. 2001;49(5):720-4.

11. Janssen HL, Leebeek FW - JAK2 mutation: The best diagnostic tool for myeloproliferative disease in splanchnic vein thrombosis? Hepatology. 2006;44(6):1391-3.

12. Janssen $\mathrm{HL}$, Meinardi JR, Vleggaar FP et al. Factor $V$ Leiden mutation, prothrombin gene mutation, and deficiencies in coagulation inhibitors associated with Budd-Chiari syndrome and portal vein thrombosis: results of a case-control study. Blood. 2000;96(7):2364-8.

13. Hollingshead M, Burke CT, Mauro MA et al. Transcatheter thrombolytic therapy for acute mesenteric and portal vein thrombosis. J Vasc Interv Radiol. 2005;16(5):651-61.

14. Senzolo M, Tibbals J, Cholongitas E et al. Transjugular intrahepatic portosystemic shunt for portal vein thrombosis with and without cavernous transformation. Aliment Pharmacol Ther. 2006;23(6):767-75.

15. Primignani M, Barosi G, Bergamaschi G et al. Role of the JAK2 mutation in the diagnosis of chronic myeloproliferative disorders in splanchnic vein thrombosis. Hepatology. 2006;44(6):1528-34.

16. Vleggaar FP, van Buuren HR, Schalm SW. Endoscopic sclerotherapy for bleeding oesophagogastric varices secondary to extrahepatic portal vein obstruction in an adult Caucasian population. Eur J Gastroenterol Hepatol. 1998;10(1):81-5.

17. Denninger $\mathrm{MH}$, Chaït $\mathrm{Y}$, Casadevall $\mathrm{N}$ et al. Cause of portal or hepatic venous thrombosis in adults: the role of multiple concurrent factors. Hepatology. 2000;31(3):587-91.

18. Valla D, Casadevall N, Huisse MG et al. Etiology of portal vein thrombosis in adults. A prospective evaluation of primary myeloproliferative disorders. Gastroenterology. 1988;94(4):1063-9. 